CERN-TH.7362/94

CTP-TAMU-37/94

NUB-TH-3098/94

\title{
NEUTRALINO EVENT RATES IN DARK MATTER DETECTORS
}

\author{
R. Arnowitt \\ Center for Theoretical Physics, Department of Physics \\ Texas A\&M University, College Station, TX 77843-4242 \\ Pran Nath \\ Theoretical Physics Division, CERN, CH-1211 Geneva 23 \\ and \\ *Department of Physics, Northeastern University, Boston, MA 02115
}

\begin{abstract}
The expected event rates for $\tilde{Z}_{1}$ dark matter for a variety of dark matter detectors are studied over the full parameter space with $\tan \beta \leq 20$ for supergravity grand unified models. Radiative breaking constraints are implemented and effects of the heavy netural Higgs included as well as loop corrections to the neutral Higgs sector. The parameter space is restricted so that the $\tilde{Z}_{1}$ relic density obeys $0.10 \leq \Omega_{\tilde{Z}_{1}} h^{2} \leq 0.35$, consistent with the COBE data and astronomical determinations of the Hubble constant. It is found that the best detectors sensitive to coherrent $\tilde{Z}_{1}$ scattering (e.g. $\mathrm{Pb}$ ) is about 5-10 more sensitive than those based on incoherrent spin dependent scattering (e.g. CaF). In general, the dark matter detectors are most sensistive to the large tan $\beta$ and small $m_{o}$ and $m_{\tilde{g}}$ sector of the parameter space.
\end{abstract}

\footnotetext{
* Permanent address
} 


\section{INTRODUCTION}

There is much astronomical evidence that more than $90 \%$ of our Galaxy, and perhaps of the universe is made up of dark matter of unknown type. In galaxies, this matter has been detected by its gravitational effects on the motion of stars and gas clouds. A large number of candidates for dark matter have been suggested both from astronomy and particle physics. In this paper we will limit our discussion to supersymmetry models with $\mathrm{R}$ parity, as they offer a natural candidate for dark matter, the lightest supersymmetric particle (LSP) which is absolutely stable. Thus the relic LSP left over from the big bang could be the dark matter present today. Further, in supergravity GUT models, for almost all the parameter space of most models, the LSP is the lightest neutralino, the $\tilde{Z}_{1}$. (The alternate possibility, that the sneutrino is lightest occurs only rarely.) Thus we will consider here only the $\tilde{Z}_{1}$ dark matter candidate, and do so within the framework of supergravity grand unification with radiative breaking.

In this paper we discuss the expected event rates for a number of dark matter detectors using the following nuclei: ${ }^{3} \mathrm{He},{ }^{40} \mathrm{Ca}{ }^{19} \mathrm{~F}_{2},{ }^{76} \mathrm{Ge}+{ }^{73} \mathrm{Ge}$, ${ }^{79} \mathrm{Ga}{ }^{75} \mathrm{As},{ }^{23} \mathrm{Na}^{127} \mathrm{I}$ and ${ }^{207} \mathrm{~Pb}$. The first two represent nuclei which are most sensitive to spin dependent incoherrent scattering of $\tilde{Z}_{1}$ by the nuclei, while the last four are increasingly sensitive to coherrent scattering. Pb could be a candidate for a superconducting detector.

A great deal of work has already been done on the question of dark matter detector rates [1-7]. We present here an analysis over the entire SUSY parameter space with $\tan \beta \leq 20$ which takes into account several important effects not generally treated before:

- Radiative breaking. Almost all previous analysis has been done within the framework of the MSSM which does not include the constraints of radiative breaking of $\mathrm{SU}(2) \mathrm{xU}(1)$. These constraints allow the determination of $\mu^{2}$ and $m_{A}$ ( $\mu$ is the $H_{1}-H_{2}$ Higgs mixing parameter, A is the CP odd Higgs boson) in terms of the other parameters. (Some 
previous analyses have varied $m_{A}$ arbitarily, obtaining spuriously large event rates.)

- As pointed out in Refs. [6,7] the heavy neutral Higgs, H, can make an important contribution to the event rates. We have included this for the entire parameter space and find that the $\mathrm{H}$ contribution relative to the light Higgs, h, can range from 1/10 to 10 times as large.

- As is well known, loop corrections to $m_{h}$ are important due to the fact that the $\mathrm{t}$ quark is heavy [8]. We have also included the loop correction to $\tilde{\alpha}$ (the rotation angle arising in diagonalizing the h- $\mathrm{H}$ mass matrix). These actually cancel much of the effects of the loop corrections to $m_{h}$.

- The COBE constraints on the $\tilde{Z}_{1}$ relic density are included. This strongly limits the region of SUSY parameter space that is allowed. In calculating these relic density constraints it is essential to include the effects of the $\mathrm{h}$ and $\mathrm{Z}$ s-channel poles [9-11] for gluinos with mass $m_{\tilde{g}} \lesssim 450 \mathrm{GeV}$. There are several effects we have not included here. Most noteworthy are that we have ommitted the possible WW, ZZ, Zh, hh final states in the $\tilde{Z}_{1}$ annihilation for the relic density calculation (which can occur when $m_{\tilde{Z}_{1}}$ gets to the upper end of its allowed spectrum i.e. $m_{\tilde{Z}_{1}} \gtrsim M_{W}$ and we have followed Refs. $[12,13]$ in calculating the relic density. We estimate that this may lead to a (25-30)\% error in the relic density, and since we have been reasonably generous in the allowed values for the relic density, we expect this will not significantly change our final conclusions. We also discuss below the sensitivity of the results to changes in the allowed region of $\tilde{Z}_{1}$ relic density.

\section{RELIC DENSITY CONSTRAINT}

The COBE data suggests that dark matter is a mix of cold dark matter, CDM, (which we are assuming here to be the relic $\tilde{Z}_{1}$ ) and hot dark matter, HDM (possibly massive neutrinos) in the ratio of $2: 1$. In addition there may also be baryonic dark matter, B, (possibly brown dwarfs) of amount $\lesssim 10 \%$ of the total. Defining $\Omega_{i}=\rho_{i} / \rho_{c}$, where $\rho_{i}$ is the mass density of the $\mathrm{i}^{t h}$ constituent and $\rho_{c}=3 H^{2} /\left(8 \pi G_{N}\right)\left[\mathrm{H}=\right.$ Hubble constant, $G_{N}=$ Newtonian 
constant] is the critical mass density to close the universe, then the inflationary scenario requires $\Sigma \Omega_{i}=1$. A reasonable mix of matter is then $\Omega_{\tilde{Z}_{1}} \simeq 0.6$, $\Omega_{H D M} \simeq 0.3$ and $\Omega_{B} \simeq 0.1$. What can be calculated theoretically is $\Omega_{\tilde{Z}_{1}} h^{2}$ where $\mathrm{h}=\mathrm{H} /(100 \mathrm{~km} / \mathrm{s} \mathrm{Mpc})$. Astronomical observations give $\mathrm{h}=0.5-0.75$. Thus we are lead to the estimate

$$
\Omega_{\tilde{Z}_{1}} h^{2} \cong 0.10-0.35
$$

Eq.(1) strongly resticts the allowed SUSY parameter space, and thus it is necessary to have a satisfactory method of calculating $\Omega_{\tilde{Z}_{1}} h^{2}$. (We will discuss below the effects of varying the maximum and minimum values of $\Omega_{\tilde{Z}_{1}} h^{2}$.) To do this, we use supergravity GUT models [14]. These models have the advantage of being consistent with the LEP results on unification of couplings at $M_{G} \simeq 10^{16} \mathrm{GeV}$ [15], and generate naturally spontaneous breaking of supersymmetry in a hidden sector. In addition, by use of the renormalization group equations (RGE), the supersymmetry breaking interactions at $M_{G}$ produce naturally spontaneous breaking of $\mathrm{SU}(2) \mathrm{xU}(1)$ at the elctroweak scale $M_{Z}$. In general, the low energy supersymmetry theory depends on only four parameters, $m_{o}, m_{\tilde{g}}, A_{t}$, $\tan \beta$, and the sign of $\mu$. Here $m_{o}$ is the universal mass of all scalar fields at $M_{G}, A_{t}$ is the t-quark cubic soft breaking parameter at the electroweak scale, and $\tan \beta=\left\langle H_{2}\right\rangle /\left\langle H_{1}\right\rangle$ where $\left\langle H_{2,1}\right\rangle$ gives masses to the (up, down) quarks.

The above may be contrasted with the MSSM (the formalism most dark matter calculations use) which possesses no theoretical mechanism for SUSY or $\mathrm{SU}(2) \mathrm{xU}(1)$ breaking and is generally parameterized by 20 aribtrary constants. In the supergravity models, all properties of the 32 SUSY particles (masses, widths, cross sections, etc.) are determined in terms of the four basic parameters and one sign. In particular, this means that $m_{A}$ and $\mu$ are so determined and are not free parameters (as usually assumed in the MSSM). Further, one finds throughout most of the parameter space the following (approximate) relations [16]: 


$$
2 m_{\tilde{Z}_{1}} \cong m_{\tilde{Z}_{2}} \cong m_{\tilde{W}_{1}} \simeq\left(\frac{1}{4}-\frac{1}{3}\right) m_{\tilde{g}},
$$

while $m_{h} \lesssim 130 \mathrm{GeV}, m_{H}^{2}>>m_{h}^{2}$ and $\tan \beta>1$. (Here, $\tilde{W}_{1,2}$ are the two charginos and $\tilde{Z}_{1,2,3,4}$ are the four neutralinos). These relations will be important in understanding the results below.

The calculation of $\Omega_{\tilde{Z}_{1}} h^{2}$ now proceeds in a standard manner. Using the RGE, we first express all SUSY masses and couplings in terms of the four basic parameters. This is done for the parameter space over the range

$150 \mathrm{GeV} \leq m_{\tilde{g}} \leq 1 \mathrm{TeV} ; 100 \mathrm{GeV} \leq m_{o} \leq 1 \mathrm{TeV} ;-2 \leq A_{t} / m_{o} \leq 6 ; 2 \leq \tan \beta \leq 20$

with a mesh $\Delta m_{o}=100 \mathrm{GeV}, \Delta m_{\tilde{g}}=25 \mathrm{GeV}, \Delta\left(A_{t} / m_{o}\right)=0.5$, and $\Delta(\tan \beta)$ $=2$ or 4 . We assume a top quark mass of $m_{t}=167 \mathrm{GeV}$, and LEP and CDF bounds are imposed on the SUSY spectrum. The $A_{t}$ range stated above exhauts the parameter space. Note that our analysis does not assume any specific grand unification group but only that it is $\alpha_{1} \equiv(5 / 3) \alpha_{Y}$ that unifies at $M_{G}$. in the early universe, the $\tilde{Z}_{1}$ is in equilibrium with quarks, leptons, etc. When the annihilation rate falls below the expansion rate, "freezeout" occurs at temperature $T_{f}$. The $\tilde{Z}_{1}$ then continues to annihilate via s-channel $\mathrm{h}$ and $\mathrm{Z}$ poles $\left(\tilde{Z}_{1}+\tilde{Z}_{1} \rightarrow h, Z \rightarrow q \bar{q}\right.$; $\ell \bar{\ell}$; etc. $)$ and t and u-channel squark and slepton poles. The relic density at present time is given by [13]:

$$
\Omega_{\tilde{Z}_{1}} h^{2} \cong 2.4 \times 10^{-11}\left(\frac{T_{\tilde{Z}_{1}}}{T_{\gamma}}\right)^{3}\left(\frac{T \gamma}{2.73}\right)^{3} \frac{N_{f}}{J\left(x_{f}\right)}
$$

where $N_{f}$ is the effective number of degrees of freedom, $\left(T_{\tilde{Z}_{1}} / T_{\gamma}\right)^{3}$ is the reheating factor and

$$
J\left(x_{f}\right)=\int_{o}^{x_{f}} d x<\sigma v>; \quad x=k T / m_{\tilde{Z}_{1}}
$$

Here $\sigma$ is the annihilation cross section, $v$ is the relative velocity and $<>$ means thermal average. Since annihilation occurs non-relativistically, 
$x_{f} \approx 1 / 20$, one may take the thermal average over a Boltzman distribution. However, as stressed in Refs. [9,10,11] one cannot generally make the nonrelativisitic expansion $\sigma v=a+b v^{2}+\ldots$ due to the presence of the narrow $\mathrm{h}$ and $\mathrm{Z}$ s-channel poles. Thus calling $\Omega_{\text {approx }}$ the evaluation using the low $v$ expansion, and $\Omega$ the rigorous result, we find for $\mu>0$ that the relation 0.75 $\leq \Omega_{\text {approx }} / \Omega \leq 1.25$ is satisfied for only $35 \%$ of the mesh points for $m_{\tilde{g}}<$ $450 \mathrm{GeV}$, but for almost $100 \%$ for $m_{\tilde{g}}>450 \mathrm{GeV}$. The reason for this can be seen from Eq. (2). One is close to an s-channel pole when $2 m_{\tilde{Z}_{1}} \approx \frac{1}{3} m_{\tilde{g}}$ is near $m_{h}$ or $M_{Z}$. Since $m_{h} \lesssim 130 \mathrm{GeV}$, this cannot happen when $m_{\tilde{g}} \gtrsim 450$ $\mathrm{GeV}$ but one is usually somewhat near either an $\mathrm{h}$ or $\mathrm{Z}$ pole when $m_{\tilde{g}} \lesssim 450$ $\mathrm{GeV}$. Thus a rigorous calculation is necessary for lower mass gluinos.

The annihilation cross section $\sigma$ can be expressed in terms of the four basic parameters $m_{o}, m_{\tilde{g}}, A_{t}$ and $\tan \beta$. Using then Eq. (4) the region in parameter space obeying the COBE constraint of Eq. (1) can be determined.

\section{EVENT RATE CALCULATION}

Dark matter detectors see the incident $\tilde{Z}_{1}$ from effects of its scattering on quarks in the nuclei of the detector. This scattering proceeds through s-channel squark poles $\left(\tilde{Z}_{1}+q \rightarrow \tilde{q} \rightarrow \tilde{Z}_{1}+q\right)$ and t-channel $\mathrm{h}, \mathrm{H}$ and $\mathrm{Z}$ poles. These are some of the crossed diagrams to the annihilation diagrams appearing in the relic density analysis. Thus to a rough approximation, one may expect the event rate to be large when the annihilation cross section is large i.e. when $\Omega_{\tilde{Z}_{1}} h^{2}$ is small. This makes results somewhat sensitive to where the lower bound on $\Omega_{\tilde{Z}_{1}} h^{2}$ is set, and we will discuss this below.

The scattering diagrams have been analysed by a number of people [1-7], and we follow the analysis of Ref. [5].* One may represent the diagrams by the effective Lagrangian

$$
\mathcal{L}_{e f f}=\left(\bar{\chi}_{1} \gamma^{\mu} \gamma^{5} \chi_{1}\right) \bar{q} \gamma^{\mu}\left(A_{q} P_{L}+B_{q} P_{R}\right) q+\left(\bar{\chi}_{1} \chi_{1}\right) C_{q} m_{q} q \bar{q}
$$

* We include an extra factor of 4 in the cross section, due to the Majorana nature of the $\tilde{Z}_{1}$, in agreement with Ref. [7]. 
where $\mathrm{q}(\mathrm{x})$ is the quark field, $m_{q}$ is its mass, and $\chi_{1}(x)$ is the $\tilde{Z}_{1}$ field. $A_{q}$ and $B_{q}$ arise from the $\mathrm{Z}$ t-channel pole and $\tilde{q}$ s-channel pole, and $C_{q}$ from the h, $\mathrm{H}$ t-channel poles and $\tilde{q}$ s-channel pole. Expressions are given for A,B,C in Ref. [5]. The first term of Eq. (6) give rise to spin dependent incoherrent scattering while the second term gives rise to coherrent scattering. There are several points to be made concerning the latter amplitude. In general, the $\tilde{Z}_{1}$ is a linear combination of two gauginos and two Higgsinos:

$$
\chi_{1}=\alpha \tilde{W}_{3}+\beta \tilde{B}+\gamma \tilde{H}_{2}+\delta \tilde{H}_{1}
$$

The $\alpha, \beta, \gamma, \delta$ can all be calculated in terms of the four basic parameters, and throughout the allowed part of the parameter space one finds

$$
\beta>\alpha, \delta>>\gamma
$$

The coefficient $C_{q}$ for the $\mathrm{h}$ and $\mathrm{H}$ poles is [17]:

$$
C_{q}=\frac{g_{2}^{2}}{4 M_{W}}\left[\left\{\begin{array}{c}
\frac{\cos \tilde{\alpha}}{\sin \beta} \frac{F_{h}}{m_{h}^{2}} \\
-\frac{\sin \tilde{\alpha}}{\cos \beta} \frac{F_{h}}{m_{h}^{2}}
\end{array}\right\}\left\{\begin{array}{l}
\frac{\sin \tilde{\alpha}}{\sin \beta} \frac{F_{H}}{m_{H}^{2}} \\
\frac{\cos \tilde{\alpha}}{\cos \beta} \frac{F_{H}}{m_{H}^{2}}
\end{array}\right\}\right] \begin{aligned}
& u-\text { quark } \\
& d-q u a r k
\end{aligned}
$$

where $F_{h}=\left(\alpha-\beta \tan \theta_{W}\right)(\gamma \cos \alpha+\delta \sin \alpha)$ and $F_{H}=\left(\alpha-\beta \tan \theta_{W}\right)(\gamma \sin \alpha-$ $\delta \cos \alpha$ ). The tree value of $\tilde{\alpha}$ (the rotation angle that diagonalizes the $\mathrm{h}-\mathrm{H}$ mass matrix) can be expressed in terms of the tree value of $m_{h}[3]$. Since loop corrections are large for the $\mathrm{h}$ particle, we have also included the loop corrections to $\tilde{\alpha}[8]$ in our calculation of $C_{q}$. Remarkably though, $\tilde{\alpha}_{l o o p}\left[\left(m_{h}\right)_{l o o p}\right]$ is generally quite close (within a few percent) to $\tilde{\alpha}_{\text {tree }}\left[\left(m_{h}\right)_{\text {tree }}\right]$. Thus $\tilde{\alpha}$ remains small i.e. $\tilde{\alpha}=O\left(10^{-1}\right.$ rad $)$. [Note, however, had one just inserted the loop correction to $m_{h}$ into the tree formula for $\tilde{\alpha}$, one would have incorrectly obtained a large value for $\tilde{\alpha}$, i.e. $\tilde{\alpha}=\mathrm{O}(1 \mathrm{rad}) !$.$] One can now see why the$ H Higgs can make a significant contribution to $C_{q}$ even though $m_{H}^{2}>>m_{h}^{2}$. For the d-quarks, the $\mathrm{h}$ term is reduced by a factor tan $\tilde{\alpha}$ relative to the $\mathrm{H}$ term. Further, the second fact in $F_{h}$ is small, either because $\gamma$ is small or 
$\sin \tilde{\alpha}$ is small. Thus for d-quarks, the $H$ contribution can range from $1 / 10$ to 10 times the h contribution, depending on the point in the parameter space. [For u-quarks, the $\mathrm{H}$ term is generally small.]

The total event rate is given by [5]

$$
R=\left(R_{\text {coh }+R_{\text {inc }}}\right)\left[\rho_{\tilde{Z}_{1}} /\left(0.3 G e V \mathrm{~cm}^{-3}\right)\right]\left[<v_{\tilde{Z}_{1}}>/(320 \mathrm{~km} / \mathrm{s})\right][\text { events } / \mathrm{kgda}]
$$

where the coherrent and incoherrent rates are

$$
\begin{gathered}
R_{c o h}=16 \frac{m_{\tilde{Z}_{1}} M_{N}^{2} M_{Z}^{4}}{\left(M_{N}+m_{\tilde{Z}_{1}}\right)^{2}} 210 \zeta_{c h}\left|M_{c o h}\right|^{2} \\
R_{\text {inc }}=16 \frac{m_{\tilde{Z}_{1}} M_{N}}{\left(M_{N}+m_{\tilde{Z}_{1}}\right)^{2}} 580 \lambda^{2} J(J+1) \zeta\left(r_{s p}\right)\left|M_{i n c}\right|^{2}
\end{gathered}
$$

Here $M_{N}$ is the nuclear mass, $\zeta\left(r_{c h}\right), \zeta\left(r_{s p}\right)$ are charge and spin form factor corrections, $\mathrm{J}$ is the nuclear spin and $\lambda$ is defined by $\left.<N\left|\Sigma \vec{S}_{i}\right| N\right\rangle=\lambda<$ $N|\vec{J}| N>$ where $\vec{S}_{i}$ is the spin of the $i^{t h}$ nucleon. ( $\lambda$ can be expressed in terms of the nuclear magnetic moment and nucleon g-factors.) $M_{c o h}$ is proportional to $C_{q}$ and $M_{i n c}$ is proportional to $A_{q}-B_{q}$, explicit formulae being given in Ref. [5].

\section{RESULTS}

Eq. (11) allows one to divide dark matter detectors into two categories: those sensitive to the incoherrent (spin dependent) scattering due to a large value of $\lambda^{2} \mathrm{~J}(\mathrm{~J}+1)$, and those sensitive to the coherrent scattering. Examples of "incohererent detectors" are ${ }^{3} \mathrm{He}$ and ${ }^{40} \mathrm{Ca}{ }^{19} \mathrm{~F}_{2}$ with $\mathrm{CaF}_{2}$ the most sensitive detector. Eqs. (11) show that $R_{\text {coh }} \sim M_{N}$ and $R_{\text {inc }} \sim 1 / M_{N}$ for large $M_{N}$, the additional $M_{N}^{2}$ factor in $R_{c o h}$ arising from the $m_{q}$ factor in Eq. (6), i.e. roughly speaking, all the quarks add coherrently to yield a $M_{N}$ factor in the amplitude. The remaining detectors considered here, ${ }^{76} \mathrm{Ge}+{ }^{73} \mathrm{Ge},{ }^{79} \mathrm{Ga}{ }^{75} \mathrm{As},{ }^{23} \mathrm{Na}{ }^{127} \mathrm{I}$ and ${ }^{207} \mathrm{~Pb}$ are all of the "coherrent" type with $\mathrm{Pb}$ being the most sensitive since it is heaviest. 
The dependence of the expected event rate on the supergravity GUT parameters is fairly complicated as each parameter enters in several places and the constraint Eq. (1) on $\Omega_{\tilde{Z}_{1}} h^{2}$ limits the parameter space. One can, however, get a qualitative picture of the parameter dependence by studying several characteristic examples . Fig. 1 shows that R decreases rapidly with $m_{\tilde{g}}$ (mainly because the $\tilde{Z}_{1}$ becomes more Bino-like). It also shows that $\mathrm{R}$ is larger for larger $\tan \beta$. (See e.g. the $1 / \cos \beta$ factor in the denominator of the d-quark part of Eq. (9); the 1/sin $\beta$ factor for the u-quark part never gets exceptionally large since $\tan \beta>1$ in the radiative breaking scenario). Finally we note that $\mathrm{R}[\mathrm{Pb}]$ is $5-10$ times larger than $\mathrm{R}\left[\mathrm{CaF}_{2}\right]$ which is also a general feature. The $\tan \beta$ dependence is shown more explicitly in Fig. 2 for the NaI and Ge detectors. (The three examples were chosen so the $\Omega_{\tilde{Z}_{1}} h^{2}$ is roughly the same at each $\tan \beta$ along each graph). The NaI curve lies higher than the Ge one for each pair since ${ }^{127} I$ is heavier than ${ }^{76} G e$.

In general, the event rate drops with increasing $m_{o}$, as one would expect since the squark mass increases with $m_{o}$, reducing the effect of the s-channel squark pole. (There are additional effects, however, as $m_{o}$ also enters in the radiative breaking equations, effecting the size of $\mu$.) Fig. 3 illustrates the general behavior for several of the detectors. The coherrent detectors, $\mathrm{Pb}$, NaI, Ge, scale almost exactly by their atomic numbers. (Fig. 3 also exhibits one of the few regions of parameter space where the $\mathrm{CaF}_{2}$ detector lies above the $\mathrm{Pb}$ detector.)

Fig. 4 exhibits the maximum and minimum event rates for the $\mathrm{Pb}$ detector (the most sensitive of the coherrent detectors) and the $\mathrm{CaF}_{2}$ detector (the most sensitive of the incoherrent detectors) as a function of $A_{t}$, as all other parameters are varied over the entire space. One sees that generally a $\mathrm{Pb}$ detector will be a factor of 5-10 times more sensitive than a $\mathrm{CaF}$ 2 detector. Other coherrent detectors have event rates that scale with the $\mathrm{Pb}$ curve in proportion to their atomic number while the ${ }^{3} \mathrm{He}$ has event rates a factor of 3 smaller than CaF. 
The above analysis has been done with $\Omega_{\tilde{Z}_{1}} h^{2}$ obeying the bounds of Eq. (1). We discuss now the effect of varying these upper and lower limits. As mentioned in Sec. III, the event rate $\mathrm{R}$ rises with decreasing $\Omega_{\tilde{Z}_{1}} h^{2}$ and this rise is rapid near $\Omega_{\tilde{Z}_{1}} h^{2} \simeq 0.1$. Further, the maximum value of $\mathrm{R}$ occurs when $m_{\tilde{g}}$ is near its minimum value. However, by the scaling relations Eq. (2) this can force $m_{\tilde{W}_{1}}<45 G e V$, and hence such parameter points are excluded by the LEP bounds. This is what causes the sharp peaks in Fig. 4, which occur when $m_{\tilde{W}_{1}}$ lies just above the LEP cut. If, for example, one increases the lower bounds on $\Omega_{\tilde{Z}_{1}} h^{2}$ to 0.15 , one finds that the maximum event rates follow the curves of Fig. 4 with the peaks cut off.

The upper bound on $\Omega_{\tilde{Z}_{1}} h^{2}$ determines the minimum event rates. This is because the minimum rates occur when $m_{\tilde{g}}$ takes on its largest value. As $m_{\tilde{g}}$ increases, so does $m_{\tilde{Z}_{1}}$ by Eq. (2). The $\tilde{Z}_{1}$ annihilation cross section then drops and $\Omega_{\tilde{Z}_{1}} h^{2}$ rises. The upper bound of Eq. (1) on $\Omega_{\tilde{Z}_{1}} h^{2}$ is found to occur when $\left(m_{\tilde{g}}\right)_{M a x} \cong 750 \mathrm{GeV}$. If one reduces the upper bound on $\Omega_{\tilde{Z}_{1}} h^{2}$ to 0.2 (which is consistent with the inflationary scenario which prefers $h \simeq 0.5$ ). Then the maximum value of $m_{\tilde{g}}$ is reduced to $*\left(m_{\tilde{g}}\right)_{M a x} \simeq 400 \mathrm{GeV}$. This then increases the minimum event rate curves of Fig. 4 by about a factor of 10.

\section{DETECTION POSSIBILITIES}

The above discussion has analysed the expected event rates for a variety of dark matter detectors over the range of parameters of supergravity GUT models. These detectors are most sensistive to the region of parameter space where $\tan \beta$ is large and $m_{o}$ and $m_{\tilde{g}}$ are small. Two types of detectors were noted: those with nuclei most sensitive to the spin dependent incoherrent scattering of the $\tilde{Z}_{1}$ (e.g. $C a F_{2}$ ), and those most sensitive to coherrent scattering (e.g. $\mathrm{Pb}$ ). In general, the best of the coherrent scatters are more sensitive than the incoherrent scatterers by a factor of 5-10. The coherrent

* Such a low mass gluino could make it accessible to detection at the Tevatron. 
scatterer event rates scale approximately with their atomic number.

Dark matter detectors currently being built plan to obtain a sensitivity to signals with $R \gtrsim 0.1$ events $/ \mathrm{kg}$ da. Future detectors may be able to obtain a sensitivity of $R \gtrsim 0.01$ events $/ \mathrm{kg}$ da. From Fig. 4 one sees that the present detectors will be able to study a small fraction of the total event rate, particularly the large $\tan \beta$ region. A more sizeable portion could become available to the next generation of detectors. However, it would appear that there will be regions of parameter space inaccessible to these types of dark matter detectors. In spite of this, the experimental study of even only a small part of the parameter space is of real importance, as such results, combined with other experiments (e.g. the $b \rightarrow s+\gamma$ decay), can together significantly limit the allowed parameter space of supergravity grand unification models. Thus recently it has been demonstrated that the experimental limits on $b \rightarrow s+\gamma$ from CLEO do indeed affect relic density analyses [18]. An analysis of the event rates with the inclusion of the CLEO constraint will be given elsewhere [19]. 


\section{FIGURE CAPTIONS}

Fig. 1 Event rates $\mathrm{R}$ vs $m_{\tilde{g}}$ for $\mathrm{Pb}$ (solid curve and $C a F$ (dashed curve) detectors, with $A_{t} / m_{o}=2.0, m_{o}=100 \mathrm{GeV}$ and $\mu<0$. The upper line

of each curve is for $\tan \beta=20$, the lower line for $\tan \beta=6$. The gap between the short branches of the curves (at $m_{\tilde{g}}=225 \mathrm{GeV}$ ) and the main branches are regions where $\Omega_{\tilde{Z}_{1}} h^{2}<0.1$.

Fig. 2 Event rates $\mathrm{R}$ vs $\tan \beta$ for $\mathrm{NaI}$ and $\mathrm{GeV}$ detectors for $m_{\tilde{g}}=275 \mathrm{GeV}$, $\mu>0$. The solid curve is for $A_{t} / m_{o}=0.0, m_{o}=200 \mathrm{GeV}$, the dashed curve for $A_{t} / m_{o}=0.5, m_{o}=300 \mathrm{GeV}$, the dash-dot curve for $A_{t} / m_{o}=$ $1.0, m_{o}=200 \mathrm{GeV}$. The upper curve of each pair of curves is for NaI, the lower for Ge.

Fig. 3 Event rates $\mathrm{R}$ vs $m_{o}$ for $A_{t} / m_{o}=0.5, \tan \beta=8, m_{\tilde{g}}=300 \mathrm{GeV}, \mu>$ 0 . The dashed curve is for $\mathrm{CaF}_{2}$, and solid curves in descending order for $\mathrm{Pb}, \mathrm{NaI}$ and $\mathrm{Ge}$.

Fig. 4 Maximum and minimum event rates vs. $A_{t} / m_{o}$ for $\mathrm{Pb}$ (solid) and $C a F$ (dashed) detectors, for $\mu<0$. All other parameters are varied over the whole space to obtain the maximum and minimum event rates. 


\section{REFERENCES}

1. M.W. Goodman and E. Witten, Phys. Rev. D31 (1985) 3059.

2. K. Greist, Phys. Rev. Lett. 62 (1988) 666; Phys. Rev. D38 (1988) 2357.

3. R. Barbieri, M. Frigini and G.F. Giudice, Nucl. Phys. B313 (1989) 725.

4. M. Srednicki and R. Watkins, Phys. Lett. B225 (1989) 140.

5. J. Ellis and R. Flores, Phys. Lett. B263 (1991) 259; Phys. Lett. B300 (1993) 175; Nucl. Phys. B400 (1993) 25.

6. M. Kamionkowski, Phys. Rev. D44 (1991) 3021.

7. M. Drees and M. Nojiri, Phys. Rev. D48 (1993) 3483.

8. J. Ellis, G. Ridolfi and F. Zwirner, Phys. Lett. B257 (1991) 83, B262 (1991) 477; H.E. Haber and R. Hempfling, Phys. Rev. Lett. 66 (1991) 1815.

9. P. Gondolo and G. Gelmini, Nucl. Phys. 360 (1991) 145; K. Greist and D. Seckel, Phys. Rev. D43 (1991) 3191.

10. R. Arnowitt and P. Nath, Phys. Lett. B299 (1993) 58; (E) B303 (1993) 403.

11. P. Nath and R. Arnowitt, Phys. Rev. Lett. 70 (1993) 3696.

12. B.W. Lee and S. Weinberg, Phys. Rev. Lett. 39 (1977) 165; D.A. Dicus, E. Kolb and V. Teplitz, Phys. Rev. Lett. 39 (1977) 168; h. Goldberg, Phys. Rev. Lett. (1983) 1419.

13. J. Ellis, J.S. Hagelin, D.V. Nanopoulos, K. Olive and M. Srednicki, Nucl. Phys. B238 (1984) 453.

14. A.H. Chamseddine, R.Arnowitt and P. Nath, Phys. Rev. Lett. 49, 970 (1982). For reviews see P. Nath, R. Arnowitt and A.H. Chamseddine, "Applied N=1 supergravity" (World Scientific, Singapore 1984); H.P. Nilles, Phys. Rep. 110 (184) 1.

15. P. Langacker, Proc. PASCOS90, Eds. P. Nath and S. Reucroft (World Scientific, Singapore 1990); J. Ellis, S. Kelley and D.V. Nanopoulos, 
Phys. Lett. B249 (1990) 441; B260 (1991) 131; U. Amaldi, W. De Boer and H. Furstenau, Phys. Lett. B260 (1991) 447; F. Anselmo, L. Cifarelli, A. Peterman and A. Zichichi, Nuov. Cim. 104A (1991) 1817; 115A (1992) 581.

16. R. Arnowitt and P. Nath, Phys. Rev. Lett. 69 (1992) 725; P. Nath and R. Arnowitt, Phys. Lett. B289 (1992) 368.

17. J. Gunion, H. Haber, G. Kane and S. Dawson, "The Higgs Hunter's Guide" (Addison-Wesley, Redwood City, 1990).

18. P. Nath and R. Arnowitt, CERN-TH-7214/94; NUB-TH.3093/94, CTPTAMU-32/94; F. Borzumati, M. Drees and M. Nojiri, KEK-TH-400, DESY 94-096, MAD/PH/835.

19. P. Nath and R. Arnowitt, CERN TH.7363/94, NUB-TH-3098/94, CTPTAMU-38/94. 
This figure "fig1-1.png" is available in "png" format from: http://arxiv.org/ps/hep-ph/9408226v1 
This figure "fig1-2.png" is available in "png" format from: http://arxiv.org/ps/hep-ph/9408226v1 
This figure "fig1-3.png" is available in "png" format from: http://arxiv.org/ps/hep-ph/9408226v1 
This figure "fig1-4.png" is available in "png" format from: http://arxiv.org/ps/hep-ph/9408226v1 